\title{
Age-Group Related Cohort Effects on the Association between Age at Menarche and Metabolic Syndrome among Korean Premenopausal Women
}

\author{
Sunmi Yoo*, Yun-Seo Hwang \\ Department of Family Medicine, Inje University Haeundae Paik Hospital, Busan, Korea
}

When we divided premenopausal women aged 20 to 55 years into two age groups (20-39 and 40-55 years) in our study, we intended to reduce the age-group related cohort effects on the association between age at menarche and metabolic syndrome (MetS). ${ }^{1)}$ The younger group (aged 20-39 years) included women born in the late 1980s, when the age at menarche decreased most rapidly in Korea. Korea has undergone rapid socioeconomic changes from the 1960s to the 1990s, so younger and older groups can be considered as different birth cohorts with distinct historical and social backgrounds. Even after dividing into two groups based on the age at the time of the survey, the mean age of the early menarche group was still lower than that of the late menarche group in both groups (29.0 years with menarche at $<12$ years versus 32.2 years with menarche at $\geq 16$ years in the women aged 20-39 years; 44.9 years with menarche at $<12$ years versus 49 .0 years with menarche at $\geq 16$ years in the women aged $20-39$ years).

MetS prevalence is well-known to increase with age, especially in women as compared with men. Recently, a prospective study with 35 European cohorts showed that the increase in MetS prevalence from the 19- to 39-year age group to the 40- to 49 -year age group was more than twofold (from $7.3 \%$ to $18.8 \%$ for the International Diabetes Federation criteria) in women and twofold (from $5.3 \%$ to $9.3 \%$ ) in men. ${ }^{2)}$ Therefore, age should be adjusted in the relationship between age at menarche and MetS, and we do not agree with the authors of the Letter to the Editor ${ }^{3)}$ that our models that included age were overadjusted. The results presented in Appendix 1 of our paper $^{1)}$ are similar to those of the recently published metaanalysis. ${ }^{4)}$ However, they ignored the age-cohort effect and made an error in applying the associations that could appear in some cohorts, for example, older women, to the whole population by analyzing various cohorts as one population.

Table 2 of our study ${ }^{1)}$ shows the mean values of each MetS component in premenopausal women according to age at menarche and age at the time of survey. Younger women with early menarche had a larger mean waist circumference than those with menarche at 12-15 years or older (mean \pm standard error, $75.98 \pm 0.62,73.08 \pm 0.31$, and $72.48 \pm 1.01$, respectively). The mean is a well-known measure of central tendency. Meanwhile, the prevalence of MetS or its individual components is the proportion of a population that displays the characteristic features of MetS at a given time. The younger women who had early and late menarche were more likely to have abdominal obesity (14.9\%, 9.9\%, and $13.7 \%$, respectively). Likewise, in the older women aged $40-55$ years, the mean waist circumference did not show significant differences among the three menarche groups, but the prevalence of abdominal obesity was highest in the early menarche group $(24.0 \%, 15.3 \%$, and $15.0 \%$, respectively). We should understand the characteristics of the statistical indexes and appropriately interpret their meaning.

\section{CONFLICT OF INTEREST}

No potential conflict of interest relevant to this article was re-

Received: May 15, 2019, Accepted: May 22, 2019

${ }^{*}$ Corresponding Author: Sunmi Yoo https://orcid.org/0000-0001-7407-8423

Tel: +82-51-797-3220, Fax: +82-51-797-2451, E-mail: syoo@paik.ac.kr 
ported.

\section{ORCID}

Sunmi Yoo: https://orcid.org/0000-0001-7407-8423

Yun-Seo Hwang: https://orcid.org/0000-0001-6258-2200

\section{REFERENCES}

1. Hwang YS, Park EJ, Choi JG, Kim HE, Park SG, Yoo SM. Relationship between age at menarche and metabolic syndrome in premenopausal women: Korea National Health and Nutrition Examination Survey
2013-2014. Korean J Fam Med 2018;39:300-6.

2. Vishram JK, Borglykke A, Andreasen AH, Jeppesen J, Ibsen H, Jørgensen $\mathrm{T}$, et al. Impact of age and gender on the prevalence and prognostic importance of the metabolic syndrome and its components in Europeans: the MORGAM Prospective Cohort Project. PLoS One 2014;9:e107294.

3. Urteaga A, Robles M, Soto A. Letter to the editor regarding the article: relationship between age at menarche and metabolic syndrome in premenopausal women: Korea National Health and Nutrition Examination Survey 2013-2014. Korean J Fam Med 2019;40:284-5.

4. Kim Y, Je Y. Early menarche and risk of metabolic syndrome: a systematic review and meta-analysis. J Womens Health (Larchmt) 2019;28: 77-86. 